\title{
Germanica
}

$53 \mid 2013$

Littérature et cinéma dans l'espace germanophone contemporain : jeux intermédiaux, modes de transfert, adaptations

\section{Transformations plus ou moins magiques. Adaptations de Der Froschkönig dans le cinéma allemand}

More or less magical transformations. The adaptations of the tale Der

Froschkönig in German films

„Nun wirst du Ruhe haben, du garstiger Frosch!“- Froschkönig-Adaptionen im

deutschen Kino

\section{Christin Niemeyer}

\section{(2) OpenEdition}

Journals

Édition électronique

URL : http://journals.openedition.org/germanica/2276

DOI : $10.4000 /$ germanica.2276

ISSN : 2107-0784

Éditeur

Université de Lille

Édition imprimée

Date de publication : 30 décembre 2013

Pagination : 89-105

ISBN : 9782913857322

ISSN : 0984-2632

Référence électronique

Christin Niemeyer, «Transformations plus ou moins magiques. Adaptations de Der Froschkönig dans le cinéma allemand », Germanica [En ligne], 53 | 2013, mis en ligne le 30 décembre 2016, consulté le 06 octobre 2020. URL : http://journals.openedition.org/germanica/2276 ; DOI : https://doi.org/10.4000/ germanica.2276 


\title{
Transformations plus ou moins magiques. Adaptations de Der Froschkönig dans le cinéma allemand
}

\author{
Christin NIEMEYER \\ Université de Caen - Basse Normandie
}

Dès la première édition des Contes de l'enfance et du foyer (Kinderund Hausmärchen) des frères Grimm, en 1812, Der Froschkönig oder der eiserne Heinrich (Le Roi Grenouille ou Henri de Fer) figure en première position. Nous le retrouvons en ouverture du recueil dans la dernière édition datée de 1857. Pourtant, une comparaison entre les versions initiale et finale montre de nombreuses modifications que les Grimm ont apportées entre-temps. Ces modifications sont, pour la plupart, des ajouts de texte successifs, qui témoignent de l'immense travail de réécriture accompli et du processus de transformation des contes entrepris par Wilhelm Grimm. Le cadet des frères Grimm travaillait les contes afin d'en augmenter la valeur morale en vue d'atteindre un public bourgeois. Son travail de réécriture, qui peut être considéré comme un véritable travail d'auteur, donna aux contes ce style typique qui conduisit André Jolles à parler du « genre Grimm ». Les modifications d'une version à l'autre se traduisaient souvent par une plus riche description des personnages et du décor, en les positionnant plus clairement dans leur environnement et en développant leur 
psychologie ${ }^{1}$. Par ce travail, la lisibilité et la logique de l'intrigue sont renforcées; des explications d'ordre quasi psychologique motivent les actions des protagonistes. Dans Der Froschkönig, les frères Grimm font notamment mieux ressortir le conflit entre père et fille concernant la promesse donnée à la grenouille, renforçant ainsi les références à la morale bourgeoise que Wilhelm Grimm souhaitait évoquer (« Il faut tenir ses promesses »).

Si ces modifications témoignent surtout du grand travail d'écriture et de réécriture fourni par Wilhelm Grimm $^{2}$, elles contredisent aussi la « légende » sur l'origine de ces contes prétendument issus du peuple dont les Grimm n'auraient été que les collecteurs ${ }^{3}$.

Le soin avec lequel les frères Grimm travaillaient et retravaillaient le conte du Froschkönig, et surtout sa position prééminente dans le recueil, laissent supposer qu'ils lui accordaient un rôle exemplaire et qu'ils voulaient en faire une sorte de «programme » au recueil qu'il ouvre.

Pourtant, même dans la version de 1857, une certaine incohérence peut surprendre, notamment par rapport aux valeurs morales du conte. Voyons ce qui se passe dans ce récit:

La princesse rencontre une grenouille qui lui ramène son jouet contre la promesse d'être son ami. Or, elle ne tient pas sa promesse et s'en va. La grenouille la poursuit au château. À l'arrivée de la grenouille au château, la princesse est obligée par son père de tenir sa promesse, elle mange et boit avec elle mais, au moment du coucher, furieuse, elle jette la grenouille contre le mur. La grenouille se transforme en prince charmant, et les fiancés partent dans le royaume du prince, accompagnés par Henri, le fidèle serviteur du prince, dont les chaînes qu'il s'était imposées lors de la métamorphose de son maître éclatent sur le chemin.

1. - C'est également la syntaxe des phrases qui a changé entre les différentes versions du Froschkönig : les phrases de la version finale sont beaucoup plus complexes au niveau syntaxique que celles de la version initiale. Lutz Röhrich compte 58 propositions subordonnées dans la version de 1857, alors qu'il n'y en avait que 27 dans celle de 1812. $C f$. Lutz Röhrich, Wage es, den Frosch zu küssen. Das Grimmsche Märchen Nummer Eins in seinen Wandlungen, Köln, Diederichs, 1987, p. 14.

2. - $C f$. les travaux fondamentaux pour la recherche sur les contes de fées entrepris par Heinz Rölleke: Heinz Rölleke, Die Märchen der Brüder Grimm. Eine Einführung, Stuttgart, Reclam, 2004 ; Heinz Rölleke, Grimms Märchen und ihre Quellen, Trier, Wissenschaftlicher Verlag, 2. Aufl., 2004.

3. - Il faut ici rappeler le rôle éminent joué par les travaux de Heinz Rölleke, qui a su démontrer que l'origine des contes dans la classe populaire est une légende créée par les frères conteurs eux-mêmes dans une perspective romantique de « retour aux sources ». En réalité, les contes ont souvent été racontés et transmis aux Grimm par des femmes issues de la grande bourgeoisie et familiarisées avec la tradition des contes français. $C f$. Heinz Rölleke (éd.), Es war einmal: Die wahren Märchen der Brüder Grimm und wer sie ihnen erzählte, Köln, Eichborn, 2011. 
Cette version du texte soulève plusieurs interrogations. Dans un premier temps, on peut se demander pourquoi la princesse semble être récompensée pour sa désobéissance en recueillant un prince charmant dans les restes de la grenouille écrasée par ses soins, alors que, pour d'autres héros et héroïnes de conte, une erreur commise peut avoir des conséquences négatives, voire funestes. (Le Petit Chaperon rouge fait un bref séjour dans l'estomac du loup pour s'être écarté du bon chemin.) Les frères Grimm, si soucieux du contenu éthique de leurs contes qu'ils les réécrivent sous bien des aspects pour en accentuer la valeur morale, auraient-ils omis la correction de cet évident manque de vertu de la part de la princesse ? Une seconde question s'impose: d'où vient le fidèle Henri de Fer et quel rôle joue-t-il dans ce conte ? Ce personnage, présent dans toutes les versions du conte, apparaît à la fin comme un deus ex machina et semble avoir pour seule fonction de vanter la fidélité d'un bon serviteur et de souligner le bonheur que lui inspire la libération du prince.

Dans le présent travail, nous n'allons ni chercher ni donner réponse à ces questions que d'autres ont soulevées avant nous ${ }^{4}$. Nous ne tenterons pas davantage une interprétation de cette prétendue incohérence. En revanche, ces deux éléments - le fait de projeter la grenouille contre le mur comme acte déclencheur d'un bonheur moralement non mérité et le rôle du fidèle Henri - nous serviront de points de départ pour notre interrogation sur les différentes adaptations cinématographiques de ce conte des frères Grimm.

Le processus de transposition d'un texte vers un film pose un certain nombre de problèmes: il faut repenser l'œuvre écrite à travers des images avec une bande sonore, des mouvements... En somme, il faut « traduire » le texte dans un autre langage, celui du cinéma. Se posent alors de nombreuses questions, et notamment celle de la fidélité au texte original: faut-il, dans la mesure du possible, adapter « mot à mot », ou bien convient-il de se permettre certaines libertés afin de respecter la logique du récit filmique qui ne correspond pas toujours à celle du récit écrit ? Ces questions ont émergé au plus tard avec la naissance du cinéma à la fin du XIX ${ }^{\mathrm{e}}$ siècle, voire bien avant, au cours de débats concernant les illustrations des contes où les problèmes du « changement de médium » (Medienwechsel) sont évoqués pour la première fois ${ }^{5}$. Comment la transposition en cet autre médium, le film, est-elle

4. - L'auteure recommande notamment la consultation des ouvrages de Lutz Röhrich et de Heinz Rölleke mentionnés ci-dessus. C'est notamment Röhrich qui livre une analyse complète de ce premier conte du recueil des Grimm : Lutz Röhrich, Wage es, den Frosch zu küssen, op. cit.

5. - Cf.notamment Fabienne Liptay, Wunder Welten.Märchen im Film, Filmstudien 26, Remscheid, Gardez-Verlag, 2004, mais aussi Emmanuelle Meunier, De l'écrit à l'écran. Trois techniques du récit : dialogue, narration, description, Paris, L'Harmattan, 2004, 
réalisée dans le cas du Froschkönig ? Comment expliquer la récompense de la princesse pour un comportement évidemment fautif ? Quant au fidèle Henri, que faire de lui ? Comment un film de conte de fées peut-il « compenser » ce récit qui comporte un certain nombre d'incohérences ? Enfin, comment peut-on étirer l'intrigue assez simple de ce conte sur une longueur de 60 minutes environ?

N'importe quelle réalisation artistique et créative s'inscrit dans le contexte historique, politique et idéologique de son époque. À l'aide des interrogations formulées ci-dessus, nous retracerons la concomitance entre le texte «classique » et le contexte respectif dans quatre adaptations allemandes du Froschkönig. Il s'agit de films produits à trois périodes différentes: le premier, tourné en RFA en 1954 par Otto Meyer, est produit par Fritz Genschow. Le deuxième a été tourné en RDA en 1988 par Walter Beck et la DEFA, alors que les deux exemples les plus récents datent de 1990 (Der Froschkönig, BRD/Tschechische Republik, Regie : Juraj Herz) et de 2008 (Der Froschkönig, BRD, Regie : Franziska Buch, dans la série Sechs auf einen Streich produite par le groupement de la Télévision allemande $A R D$ ). Comment les différents réalisateurs résolvent-ils les problèmes que pose le texte original ?

\section{Der Froschkönig, RFA 1954}

\section{(réal. Otto Meyer, production : Fritz, Genschow Film, 83 minutes)}

Ce film est la première adaptation allemande d'après-guerre du conte Der Froschkönig oder der eiserne Heinrich ${ }^{6}$. Elle a été tournée en grande partie dans le parc du Château de Charlottenburg à Berlin. $\mathrm{Si}$ un certain Otto Meyer est le réalisateur de ce film, il semble plus intéressant de se pencher sur l'entreprise de production responsable de ce film : la Fritz Genschow Film. Le réalisateur et producteur Fritz Genschow était la figure de proue de la production des films de contes de fées d'après-guerre en RFA. Son engagement dans le cinéma pour enfants a pourtant un antécédent. Genschow tourna son premier film de conte de fées en 1937: une adaptation de Rotkäppchen und der böse Wolf (Le petit Chaperon rouge et le méchant loup), qui affiche des références très explicites au national-socialisme. Comme le montre le chercheur Ron Schlesinger, Genschow et de nombreux autres réalisateurs de cette époque adaptèrent leurs scénarios basés sur les contes de fées - de préférence des frères Grimm - au contexte politique du national-

Jeanne-Marie Clerc et Monique Carcaud-Macaire, L'adaptation cinématographique et littéraire, Paris, Klincksieck, 2004, et Joachim Paech, Literatur und Film, Stuttgart/ Weimar, Metzler, 1997.

6. - Il existe une version du Froschkönig de 1940, tournée par Alf Zengerling. Malheureusement, cette version, certainement hautement intéressante en raison de son contenu idéologique, ne nous était pas accessible au moment de la rédaction de cet article. 
socialisme afin d'en faire des outils de la propagande nazie ${ }^{7}$. Ainsi, le chasseur, dans la version du Petit Chaperon rouge de Fritz Genschow (1937), arbore fièrement la croix gammée sur le revers de sa veste. Dans la mise en scène de Hubert Schonger en 1940, Le petit soldat de plomb (Der standhafte Zinnsoldat), inoffensif dans la version littéraire écrite par Andersen, réunit ses troupes et détruit un troll dans une guerre impitoyable. Il donne ainsi l'exemple à suivre : l'Allemagne, tout comme le petit soldat, ne peut se défendre contre ses ennemis (les trolls) que par la guerre totale ${ }^{8}$. Après la Seconde Guerre mondiale, Genschow, Alf Zengerlein et Hubert Schonger continuent à réaliser et à produire des films de contes de fées. Entre la fin des années quarante et la fin des années cinquante, on enregistre même une production très dynamique de films de contes de fées ${ }^{9}$. Toutefois, la plupart de ces adaptations sont d'une qualité très médiocre.

Quant à la version du Froschkönig produite par Genschow et réalisée par Otto Meyer en 1954, le Lexikon des Internationalen Films en ligne la qualifie comme suit: il s'agirait «d'un conte des frères Grimm dans une adaptation des années 1950 destinée aux enfants et avec un jeu d'acteurs remarquable ${ }^{10}$. Voyons cela dans le détail:

Le film commence par l'historique de la transformation en grenouille. Le spectateur rencontre un jeune prince au nom prometteur d'Edelmut, dont le père est gravement malade. Le prince part à la recherche du remède destiné à guérir son père. Il est accompagné de son

7. - Dans ce contexte, il est intéressant de rappeler que le film d'animation était également souvent au service de la propagande nazie. Enthousiaste devant les productions de Walt Disney, Hitler avait commandé des films d'animation du même genre qui devaient servir ses buts de propagande. Douze de ces films d'animation tendancieux sont réunis dans le coffret de DVD Geschichte des deutschen Animationsfilms publié, en 2011, par absolutMedien et sous la direction d'Ulrich Wegenast. Y sont représentées également des productions de Schonger. $C f$. entre autres http://www.fr-online.de/ kultur/trickfilme-zur-ns-volksaufklaerung/-/1472786/9550624/-/index.html (consulté le $15 / 08 / 2013, \mathrm{CN}$ ).

8. - Cf. Ron Schlesinger, "Heil dem gestiefelten Kater! » - NS-Propaganda in Märchenfilmen zwischen 1933 und 1945. À consulter sur Internet à l'adresse: http:// leviathan0712.posterous.com/heil-dem-gestiefelten-kater-ns-propaganda-in (consulté le 17/08/2011).

9. - Pour en savoir plus sur le film de contes de fées en RFA, voir la thèse de doctorat de Christoph Schmitt, chercheur à l'Université de Rostock : Christoph Schmitt, Adaptionen klassischer Märchen im Kinder- und Familienfernsehen. Eine volkskundlichfilmwissenschaftliche Dokumentation und genrespezifische Analyse der in den achtziger Jahren von den westdeutschen Fernsehanstalten gesendeten Märchenadaptionen, mit einer Statistik aller Ausstrahlungen seit 1954, Frankfurt am Main, Haag + Herchen, 1993 (Studien zur Kinder- und Jugendmedienforschung; 12) .

10. - http://www.zweitausendeins.de/filmlexikon/?wert=25579\&sucheNach=ti tel, consulté le 19 avril 2013, "Grimmsche[s] Märchen in einer kindgerechten und schauspielerisch beachtlichen 50er-Jahre-Fassung » [Trad. CN]. 
fidèle serviteur Heinrich qui, lui, est chargé par le vieux roi de garder le trône jusqu'à la majorité du prince et de protéger ce dernier. Lorsque les deux chevaliers arrivent chez la méchante comtesse détenant la potion magique, celle-ci refuse son aide pour guérir le roi. Le prince s'emporte et la sorcière le transforme alors en grenouille. Malgré les implorations de Heinrich la priant de conjurer le sort, elle refuse, mais explique que seul le baiser d'une jeune fille vertueuse pourra délivrer le prince. Puis changement de décor: cinq ans plus tard, on voit la fille cadette du roi, prénommée Susanne, qui fête ses 16 ans. Depuis peu, elle entend des appels au secours venant du lac. Malheureusement, elle est la seule à les entendre. Mais ses deux sœurs croient se souvenir que ce fut précisément auprès de ce lac que le prince Edelmut avait été transformé en vilaine grenouille. Bien que les deux sœurs aînées, en quête d'un bon mariage, cherchent partout la grenouille, elles ne la trouvent pas. En revanche, la cadette continue d'entendre des voix et parvient même, un jour, à parler avec l'animal. Lorsqu'elle lui ramène sa balle d'or qui était tombée à l'eau, la jeune princesse invite la grenouille au château et lui promet d'être sa compagne. Dans une longue scène tendrement filmée, la grenouille se régale à la table du roi avant de se retirer dans la chambre avec la princesse. C'est même la princesse elle-même qui l'emmène dans les plis de sa robe. Sur le chemin, la princesse est bousculée et croit avoir perdu la grenouille. Triste de ne pouvoir tenir sa promesse, elle se confie à son valet Johann. Celui-ci, prévoyant et fidèle serviteur de la douce princesse, avait sauvé la grenouille et la lui ramène à présent. Arrivée dans la chambre, la grenouille demande aussitôt un baiser à la princesse. En prononçant elle-même les mots clés "Was man versprochen hat, muss man auch halten », Susanne s'empresse de l'embrasser ${ }^{11}$. La magie opère, le prince Edelmut est délivré et se présente à la princesse. Le film, auparavant tourné en noir et blanc, passe alors en couleur ${ }^{12}$. Le prince rentre aussitôt dans son royaume et promet d'être de retour sous huit jours afin de demander Susanne en mariage. Lorsque le prince revient, c'est le fidèle Heinrich qui annonce son retour et exprime sa joie de revoir le trône du royaume de nouveau occupé. Le prince demande Susanne en mariage et la ramène dans son royaume. Suit la scène connue où les chaînes que le fidèle Heinrich avait forgées autour de son cœur afin de se rappeler son vœu de fidélité envers son maître se brisent enfin. Cette scène est fidèlement reprise du récit des frères Grimm.

Ce film a opté pour une adaptation très fidèle du modèle littéraire - à une exception près. En ajoutant un antécédent, deux «blancs » du récit

11. - Dans la version des frères Grimm, ces mots sont prononcés par le roi rappelant à sa fille les règles d'un comportement correct.

12. - On se réfère à la version DVD sortie par ICESTORM. 
original trouvent explication: le spectateur apprend la cause de la transformation en grenouille ainsi que l'origine et le rôle du fidèle Heinrich. Quant au violent lancer de la grenouille contre le mur, il a été supprimé et remplacé par son contraire : un baiser, dont le modèle semble clairement être le monde merveilleux de Disney ${ }^{13}$. En effet, le caractère de la princesse Susanne tel que présenté dès le début du conte n'aurait pas correspondu à un acte aussi cruel que de projeter la grenouille contre le mur. La douceur de cette jeune fille modèle, accentuée par le caractère très peu aimable de ses sœurs aînées, constitue un pilier de cette adaptation où les bons ne sont que bons et les méchants sont gentiment punis à la fin.

Quant à Heinrich, c'est sa fidélité exemplaire qui est soulignée par le rôle important qu'il joue dans le récit-cadre du film. On retrouve le reflet de cette fidélité (par ailleurs une « vertu allemande » très appréciée par les nationaux-socialistes et rappelant la fameuse Nibelungentreue) dans le personnage de Johann, domestique de la princesse Susanne sans lequel la délivrance du prince n'aurait pas été possible.

\section{Froschkönig, RDA 1987}

\section{(réal.: Walter Beck, 67 minutes)}

Froschkönig, une production des studios de la DEFA en RDA, réalisée par Walter Beck, se distingue déjà par son titre : il ne renonce pas seulement à l'évocation du fidèle Henri, mais il supprime aussi l'article ${ }^{14}$. Par l'omission du déterminatif, le film Froschkönig se met sciemment à distance du récit original, et le groupe de travail « Johannisthal » 15 le met en scène « librement adapté du conte des frères Grimm » (frei nach dem Märchen der Brüder Grimm).

Cette version de Walter Beck, réalisateur d'un grand nombre de films de conte de fées de la DEFA, rencontre un accueil mitigé en RDA. Tandis que certains critiques lui reprochent d'illustrer tout au plus le

13. - Celui-ci s'était déjà servi du pouvoir universel et magique du baiser romantique pour le tournage de plusieurs de ses grands succès de film d'animation, comme Blanche-Neige et les sept nains (1937) et Cendrillon (1950).

14. - Le réalisateur Walter Beck, dans une lettre personnelle à l'auteure de cet article, insiste sur la suppression de l'article comme acte et volontaire et réfléchi.

15. - Les studios de la DEFA étaient divisés en différents groupes de travail. Le nom du groupe « Johannisthal » était une référence au quartier berlinois dans lequel était située la société Johannisthaler Filmanstalt GmbH depuis 1920. Elle produisit, entre autres, des classiques du cinéma allemand tel que Nosferatu le vampire de Friedrich Wilhelm Murnau (1922). Avec l'arrivée du cinéma parlant, ces studios furent repris par la société Tobis-Filmkunst GmbH qui, elle-même, fut reprise par la DEFA après la Seconde Guerre mondiale et lors de la mise en place des studios est-allemands en 1946. Cf. Wolfgang Jacobsen, Babelsberg. 1912-1992. Ein Filmstudio, Berlin, Stiftung Deutsche Kinemathek, 1992 ; Günter Jordan, Film in der DDR. Daten, Fakten, Strukturen, Potsdam, filmmmuseum Potsdam, 2009. 
danger que présente toute adaptation d'un conte des frères Grimm et se moquent de la princesse qui ferait une formidable « fille de fermier » (Bauerntochter) ${ }^{16}$, d'autres félicitent le réalisateur et son équipe d'avoir réussi une belle « réécriture » du conte, sans pour autant mettre à l'écart ses origines. Les critiques positives soulignent surtout le scénario conséquent qui a annulé la contradiction du récit original en proposant une fin à la fois logique, merveilleuse et humaniste ${ }^{17}$.

En effet, cette adaptation est, pour une large part, une extrapolation du récit des frères Grimm. L'intrigue du conte ne couvre que les cinq premières minutes du film. Puis, au lieu d'épouser le prince charmant qui vient de tomber si merveilleusement du mur contre lequel elle voulait écraser la grenouille, la princesse, ici prénommée Henriette, apprend une triste nouvelle : ex-grenouille, ce jeune homme auquel elle fait aussitôt les yeux doux n'est point délivré. Comme elle n'a pas tenu sa promesse de manger, de boire et de dormir avec la grenouille, le prince doit s'en aller au bout du monde, dans un endroit où il n'est que tristesse. Puis, la majeure partie du film est consacrée à la quête de la jeune fille pour retrouver son prince charmant. Elle part au bout du monde, se travestit en garçon, se fait engager en tant que commis et valet afin de tenir enfin ses promesses et de délivrer son bien-aimé. La « contradiction » du conte qui fait profiter la princesse d'un bonheur non mérité est ici dissoute par l'extrapolation et la modification de la fin. Par la même occasion, Beck trouve aussi une solution pour « expliquer » le personnage du fidèle Henri, absent du titre. Lorsque la princesse Henriette arrive à la « citadelle de la désolation » (Zitadelle der Trostlosigkeit) où son prince vit isolé du monde, elle apprend que nulle femme ne peut y entrer. Afin de relever tout de même le défi, Henriette se sépare de sa chevelure, se déguise en garçon et se fait embaucher à la

16. - Anonym, Gebrüder Grimm brav und bieder, dans : « Mitteldeutsche Neueste Nachrichten » (Leipzig), 2. Juli $1988:$ « [...] In einer gedrechselten Kunstsprache konversieren blutleere Gestalten in stillosen Puppenstuben-Interieurs. Der Frosch ist eine artig gestylte Aufblaskreation und Jana Mattukat wäre eine prima Bauerntochter. Unerbittlich fotografiert die Kamera diese Fehlbesetzung einer Prinzessin, während der veränderte Fabelfaden, brav und bieder inszeniert, vor sich hin dröselt. [...] Walter Becks Film nimmt sich wie eine Warnung aus: Grimmsche Märchen sind verdammt sperrige Adaptionsstücke. »

17. - Heinz Hoffmann, Wunderbare Wandlung durch Liebeskraft. Das Märchen vom Froschkönig filmisch neu erzählt, dans : « Nationalzeitung » (Berlin), 5. Juli 1988 : «Walter Beck fand in der Szenaristin Brigitte Bernert und im Dramaturgen Gert Gericke aufmerksame Partner, denen d[...]er Widerspruch und die Zweiteilung des Märchens nicht entgangen waren. Sie erzählen das Märchen neu, ohne seinen Ursprung auszugrenzen. Sie erzählen in poetischer Erweiterung den moralischen Reifeprozess und die wundersame Wandlung der Königstochter Henriette durch die Kraft der Liebe [...]. So denken sie das moralische Anliegen des Grimmschen Märchens logisch zu Ende und setzen an die Stelle des Widerspruchs eine klare geistige Orientierung auf den zutiefst humanistischen Anspruch menschlicher Bewährung. [...]» 
citadelle en tant que domestique. Elle se fait appeler Heinrich, version masculine de son vrai nom. C'est précisément ce personnage qui, à la fin du film, donnera la fameuse réplique à l'exclamation du prince : «Heinrich, der Wagen bricht. »; « Nein, Herr, der Wagen nicht, es ist ein Band von meinem Herzen, das da lag in großen Schmerzen...». La délivrance du prince est ainsi présentée comme étant aussi celle de sa libératrice. Il y a enfin un vrai partage; la promesse initiale, point déclencheur et pivot de l'intrigue, est tenue.

Walter Beck trouve des solutions très intéressantes pour combler les « blancs » du récit des frères Grimm: si lancer la grenouille contre le mur constitue l'acte de colère d'une jeune femme rebelle, la suite du récit filmique lui apprend la maturité. Cette adaptation semble illustrer la doctrine pédagogique selon laquelle les promesses sont à tenir, mais elle apprend aussi au jeune spectateur que le bonheur n'est pas servi sur un plateau et qu'on ne saurait y accéder sans efforts. Y compris dans l'univers du conte ${ }^{18}$. Cette princesse au visage très moderne doit mériter son bonheur, et elle l'obtient uniquement par la force de son caractère et de son amour. Cependant, une telle version n'est pas dénuée d'inconvénients : Walter Beck a, par exemple, dû sacrifier certains aspects romantiques du merveilleux, décontenançant ainsi certains critiques. Sa version, plutôt sombre, ne s'adresse probablement pas d'abord à un public d'enfants, mais elle constitue surtout une véritable réécriture proposant une approche moderne, sans pour autant trahir les frères Grimm, dont le récit est entièrement repris dans la première partie du film.

\section{Der Froschkönig, RFA 1990}

\section{(réal. : Juraj Herz, 90 minutes)}

Der Froschkönig, dans sa version de 1990, a été projeté pour la première fois lors du festival Kinderfilmtage Ruhr en novembre 1991 et a été diffusé à la télévision publique (ZDF) le 25 décembre 1991. Le Lexikon des Internationalen Films en ligne le qualifie sévèrement de « divertissement globalement ennuyeux dont les photos sont trop lisses et trop propres pour transmettre l'essence du conte ${ }^{19} »$.

18. - En effet, Henriette insiste, à plusieurs reprises, sur le fait que, selon les codes du conte, le prince devrait être délivré. Apprenant que tel n'est pas le cas, elle s'écrie même qu'il ne devrait pas en être ainsi. Le spectateur a l'impression que l'héroïne elle-même connaît le fonctionnement «normal » d'un conte et qu'elle dénonce ici la dérogation à la règle. Ce jeu avec les codes du conte est une caractéristique que l'on retrouve dans plusieurs adaptations des contes de fées tournées à la DEFA.

19. - http://www.zweitausendeins.de/filmlexikon/?sucheNach=titel\&wert=27554; consulté par l'auteure le 19 mai 2013: «Weitgehend langweilige Unterhaltung, deren Bilder allzu sauber und eindeutig sind, um die Essenz des Märchens [...] einfangen zu können. » [Trad. CN] 
Ce film complète l'intrigue des frères conteurs par un scénario assez complexe mêlé à des éléments plus classiques :

Un roi a trois filles. Selon la prophétie d'une fée, son royaume sera inondé par un flot de larmes si les trois princesses ne se marient pas dans leur ordre d'âge à treize mois d'écart. L'aînée est désormais mariée, le mariage de la deuxième princesse est imminent. À cette occasion, un grand nombre de nobles arrivent pour les festivités, parmi eux un jeune prince qui a brisé le cœur de plus d'une femme. Ayant appris que la cadette des trois princesses est très belle, il a hâte de faire sa connaissance. Or, cette jeune fille ne s'intéresse pas au mariage, préférerait être un homme et passe son temps à rôder dans les forêts en habit de garçon. Son comportement ne correspond guère à celui d'une princesse. Lorsque le prince arrive à la cour, la femme de chambre de la princesse se permet une plaisanterie et avertit le valet du prince de la grande laideur de sa maîtresse. Celui-ci rapporte aussitôt cette information à son ami et maître. Lorsqu'il l'aperçoit enfin, le prince est donc passablement surpris de voir que la princesse n'a rien de laid. Dans une forêt, il essaie de l'embrasser, mais ne reçoit qu'une gifle en retour, et la princesse s'en va. Le prince s'assoit auprès d'une fontaine magique dans la forêt et s'endort. Dans son rêve, la fée de la fontaine lui apparaît et l'informe qu'elle le transformera en grenouille s'il venait à briser un autre cœur ou s'il devait être la cause d'une nouvelle affliction. Une fois transformé, seul l'amour d'une femme fidèle pourra le délivrer. Croyant avoir rêvé, le prince ne prend pas ces menaces au sérieux. En attendant, la princesse vient de se rendre compte qu'elle est éprise de ce prince hardi et change de comportement à son égard. Lors du bal, et à la surprise de toute la cour, elle apparaît dans une magnifique robe blanche et permet au prince de danser avec elle. Seulement, une comtesse, elle-même amoureuse du prince, est jalouse d'elle. Le prince réessaie d'embrasser la jeune fille, qui prend de nouveau la fuite. Déçu et étrangement attiré par la fontaine dans la forêt, il y retourne et rencontre la comtesse jalouse qui le provoque par des paroles osées. Retombant dans ses travers de briseur de cœur, le prince finit par l'embrasser. Or, la princesse, qui arrive à ce moment même près de la fontaine, voit ce baiser et s'en va en pleurant. La magie de la fontaine prend alors effet, le prince est aussitôt transformé en grenouille. Alors qu'il ne retourne pas au château, la princesse se reproche son comportement brusque envers lui, mais reste convaincue qu'il reviendra un jour au château. Elle refuse dès lors de se marier avec d'autres prétendants. Les treize mois s'écoulent, au terme desquels elle devrait se marier pour ne pas exposer le royaume au déclin. Juste avant la date de la terrible échéance, elle se promène près de la fontaine dans la forêt où sa balle d'or tombe à l'eau. Entendant les pleurs de la jeune fille, une grenouille apparaît et lui promet de lui rapporter sa balle à condition de manger et de boire 
avec elle, de dormir dans son lit et de recevoir un baiser de sa part. La princesse donne sa parole, récupère sa balle et s'en va. Or, la grenouille - nul autre, bien sûr, que notre prince malheureux - la suit dans un parcours passablement aventureux. Les treize mois étant écoulés, le peuple angoissé demande le mariage forcé de la princesse afin de sauver le pays. C'est à ce moment précis qu'apparaît la grenouille au château, demandant que la princesse tienne sa promesse. Pour le bien du peuple, elle accepte, mange et boit avec lui et l'emmène dans sa chambre. Elle s'efforce même de lui donner le baiser qu'elle demande. À ce momentlà, minuit sonne, la grenouille est retransformée en prince, le peuple est satisfait et un feu d'artifice accueille le jeune couple.

Dans cette version pourtant (trop) riche en action, aucune mention n'est faite du personnage du fidèle Henri. La princesse ne projette pas non plus la grenouille contre le mur, au contraire, elle respecte sa promesse en espérant sauver le pays. Elle pose un acte altruiste en donnant un baiser à une créature qui la répugne, en s'effaçant au profit du bien-être de tous. On peut y voir l'acte d'une personne qui prend enfin ses responsabilités, d'une jeune fille qui a grandi - ou celui d'une jeune femme, indépendante au départ et qui finit par se soumettre. Toujours est-il que le film procède à une réécriture plus ample que ses prédécesseurs en modifiant les scènes phares du conte. Ainsi, la violence envers la grenouille est transformée en baiser. Dans le même temps, la princesse mégère est apprivoisée: elle qui refusait d'embrasser un joli prince donne un baiser à une vilaine grenouille.

Le personnage de Heinrich est supprimé, et d'autres personnages secondaires (non présents dans le récit littéraire) servent de déclencheurs de l'intrigue (la femme de chambre Rose, le valet du prince). Dans l'ensemble, ce film, d'une durée considérable (90 minutes) pour une production destinée à un jeune public, meuble une intrigue globalement faible, quoique divertissante, par quelques éléments empruntés au genre de la comédie romantique ou à d'autres contes de fées, sans lien avec l'intrigue du conte original. Mais il ne propose pas de véritable interprétation ni d'explication des (prétendues) incohérences du récit Der Froschkönig.

\section{Der Froschkönig, Deutschland 2008}

(réal. : Franziska Buch, 60 minutes : Sechs auf einen Streich, ARD)

À l'inverse, cette adaptation très récente respecte scrupuleusement le format d'un film jeune public en se contentant d'une intrigue de 60 minutes. Elle a été tournée dans le cadre du projet Sechs auf einen Streich du groupement public de neuf radiodiffuseurs régionaux allemands ARD. Depuis 2008, ce groupement de chaînes publiques a produit plusieurs séries d'adaptations de films de contes 
de fées classiques des frères Grimm et de Hans Christian Andersen. Der Froschkönig fait partie de la première saison, tournée en 2008. Les concepteurs de la série, soucieux de tourner des adaptations plus modernes tout en respectant le merveilleux des récits classiques et en laissant de côté tout élément anachronique, ont été nominés au prestigieux prix Adolf-Grimme en 2010. De nouvelles productions sont prévues pour une diffusion télévisée à Noël 2013. Les 26 productions tournées jusqu'à ce jour se distinguent surtout par un casting exceptionnel d'acteurs allemands connus et populaires tel que Richy Müller (Heinrich) et Friedrich von Thun (le roi) ainsi que de jeunes espoirs parmi les acteurs allemands tel que Sidonie von Krosigk dans le rôle principal de cette production. Comment se déroule donc l'intrigue de ce Froschkönig qui cherche à réconcilier le moderne et le classique ? Voici un bref résumé du récit filmique:

La princesse Sophie a 18 ans, et le royaume est en fête. Mais les nombreux présents et les festivités pompeuses mises en scène selon la mode rococo ne parviennent guère à dissimuler le malheur qui se profile à l'horizon: le royaume est au bord de la faillite. Le roi veut donc obliger sa fille à épouser le prince (Friedrich) du royaume voisin pour sauver le pays. Sophie, jeune fille sauvage et indépendante, rejette cette idée jusqu'à ce que la fameuse balle d'or fasse évoluer les choses. En effet, dans cette version, la balle d'or représente l'héritage spirituel de sa mère décédée dans des circonstances étranges. Grâce à elle, Sophie devra apprendre à suivre son cœur plutôt que sa raison. Et puis la balle tombe dans l'étang. La grenouille, impertinente, s'introduit dans le château et oblige la jeune fille à partager son repas et sa chambre. C'est dans la chambre où la grenouille veut gagner le lit de la jeune fille que la magie de la balle opère: la princesse jette la grenouille contre le mur et délivre le prince de son enchantement. Et, surtout, elle s'oppose à la volonté de son père en refusant le mariage de raison et en suivant son propre chemin.

Ici, c'est donc l'esprit de contestation, l'opposition saine à une autorité, qui est retenue : l'incohérence morale est transformée en comportement naturel et donc juste. La jeune fille rebelle de cette version doit se révolter pour s'arracher à la tutelle de son père. Grandir, c'est s'opposer, semble dire cette version très moderne du conte. Projeter la grenouille contre le mur n'est donc aucunement un acte immoral, mais représente un comportement tout à fait naturel de la part d'une jeune fille en passe de devenir adulte.

Cela semble être dans l'intention des producteurs, comme le prouve le dossier de presse de la production:

Und die Moral von der Geschicht'? 
Redakteurin Margret Schepers: Wir alle kennen ja den Spruch des Königs: Was man versprochen hat, muss man auch halten! Aber nur weil die Prinzessin sich empört und den Frosch gegen die Wand wirft - und damit gegen das Verdikt des Vaters verstößt -, kann der Frosch erlöst werden. Also: Folge deinem Herzen - auch in der Wut und in den Widersprüchen.

Contrairement à la version du début des années 1990, cette princesse plus récente est donc une jeune femme dotée d'un caractère bien trempé, conçue comme le modèle d'une jeune fille moderne qui suit son propre chemin. La doctrine de la promesse à tenir est donc caduque. Il n'est plus nécessaire d'expliquer pourquoi la violence envers la grenouille est récompensée car, ici, cet acte n'est pas considéré comme immoral, mais comme une opposition naturelle et nécessaire au bon développement d'une jeune femme.

Quant au personnage du fidèle Heinrich, il est introduit progressivement et de manière mystérieuse. Dès le début du film, on voit évoluer un personnage taciturne muni de chaînes autour de la poitrine partir à la recherche de quelqu'un. Il approche de plus en plus du château et attend que son maître soit délivré de son sort. Comme une ombre, il suit son maître et anticipe son destin pour se tenir prêt au moment où celui-ci aura de nouveau besoin de ses services. D'une certaine manière, il peut être considéré comme le pendant de la figure de la mère de la princesse incarnée par la balle d'or et représentant le destin de la fille et la protection dont celle-ci jouit. Ce personnage n'a, certes, pas une grande importance pour la progression de l'intrigue à proprement parler, mais son apparition dès le début du conte a pour fonction de combler le «blanc » laissé par le récit original.

\section{Bilan}

Ce petit aperçu des adaptations du conte numéro un des frères Grimm montre que les prétendues faiblesses de ce conte, avec une intrigue dont la morale apparaît pour le moins étrange et des personnages qui semblent surgir de nulle part, s'avèrent être des richesses pour les réalisateurs. En effet, ce sont souvent ces «blancs » du récit original qui donnent lieu à une réinterprétation ou à une véritable réécriture du conte avec le langage du cinéma. Les aspects et les approches choisis varient considérablement selon l'époque de production.

Dans la version des années 1950 en RFA, la vertu des protagonistes et surtout celle de la jeune princesse figure au centre du film et détermine son intrigue. Par une réalisation très moralisatrice du conte portée par un ensemble de personnages exemplaires et vertueux, les réalisateurs se réfèrent clairement au récit original des frères Grimm, à l'héritage littéraire allemand et au système de valeurs que les auteurs 
souhaitaient transmettre par leurs contes. Par l'évocation des vertus traditionnelles - une princesse est belle, douce et fidèle ; un valet suit fidèlement son maître jusqu'au bout du monde et même plus loin, au risque de sa propre vie -, le film rappelle d'anciennes valeurs que l'on qualifiait encore de typiquement allemandes quelques années plus tôt. Cette continuité avec le passé ne peut guère surprendre si on rappelle le nom du producteur de ce film, Fritz Genschow. Sans pour autant permettre de repérer la moindre allusion à l'idéologie nazie dont les anciens films de Genschow regorgent, cette version du Froschkönig peut être qualifiée de traditionaliste à souhait.

Cette orientation se manifeste précisément dans la mise en œuvre des «blancs » que nous avons évoqués au début de ce texte : la question de la violence envers la grenouille est éliminée et remplacée par une modification radicale de cette bribe d'intrigue; l'apparition du fidèle Heinrich - qui ne pourrait guère être plus fidèle que dans cette réalisation - souligne l'importance de la valeur qui est au centre de cette adaptation: la fidélité.

Les versions plus récentes accentuent d'autres éléments.

L'adaptation de la DEFA tournée à la toute fin de la RDA, lorsque les directives politiques pour un cinéma socialiste se faisaient de moins en moins sentir, souligne surtout les valeurs morales universelles, ici résumées par la phrase-clé du conte : «Was du versprochen hast, musst du auch halten. » Jeter la grenouille contre le mur apparaît ici comme un acte condamnable parce qu'il coïncide avec le refus de tenir une promesse donnée. C'est précisément ce comportement fautif qui lancera l'intrigue à proprement parler. La princesse de cette version a une leçon à apprendre: rien n'est servi sur un plateau; il faut agir pour atteindre le bonheur. Dans le contexte d'une RDA d'avant la chute du Mur où l'incertitude sur l'avenir du pays et des valeurs socialistes caractérisait le travail de tout artiste, ce film, dont la couleur principale est le gris, se lit parfois comme un requiem. Les dirigeants de la RDA avaient réclamé, pendant les années 1950, une « interprétation progressiste » pour toute réalisation cinématographique des contes de fées. Ce qui voulait dire: donner des «modèles » (Leitbilder) aux jeunes spectateurs afin d'en faire des socialistes à part entière. Pendant les années 1980, cette orientation socialiste des films pour enfants se perd de plus en plus et cède la place à une vision plus universelle des valeurs du conte de fées. Le périple de la princesse Henriette illustre cette nouvelle voie en RDA. Les réalisateurs ne critiquent en rien le parti, les dirigeants de leur pays ou la situation désastreuse dans laquelle se trouvait la RDA à la fin des années 1980 - comme cela a pu se faire dans certains films de contes de fées des années 1960 et 1970 . S'il y a risque, celui-ci ne ressortit qu'à la mise en scène dans les tons gris et l'extrapolation audacieuse de l'intrigue, visant à donner au récit une cohérence morale. En revanche, 
le film de Walter Beck propose l'interprétation la plus audacieuse et la plus moderne du personnage du fidèle Heinrich en l'associant au destin de la princesse. Henriette devient Heinrich. La beauté féminine et la séduction ne servent à rien pour le défi que cette princesse doit relever. Ce n'est que la fidélité envers sa promesse initiale qui compte. Le Heinrich du conte étant l'incarnation de cette fidélité, Henriette se transforme donc à son tour: elle devient Heinrich et change de caractère en reconnaissant l'importance de la fidélité. Les limites imposées par les rôles traditionnellement attribués aux princes et aux princesses et, par conséquent, celles des codes du genre sont repoussées dans ce récit, si bien que cette version propose une véritable réinterprétation du conte avec une modernité qui n'a pas été égalée jusqu'à nos jours.

L'adaptation du début des années 1990, une co-production avec des studios tchèques ayant une grande et remarquable expérience dans la production des films de contes de fées, ne s'attarde pas à expliquer les prétendues incohérences et ne conserve que les grandes lignes du conte. Les années 1990 sont la décennie où la comédie romantique fête ses plus grands succès: Pretty Woman (réal.: Garry Marshall, 1990), Bodyguard (réal. : Mick Jackson, 1992), Nuits blanches à Seattle (réal. : Nora Ephron, 1993) deviennent des films cultes pour la génération des jeunes déstabilisés par les bouleversements politiques qui secouent l'Allemagne et tout le bloc de l'Est au début des années 1990. Avec son chassé-croisé d'histoires d'amour et de malentendus, Der Froschkönig de Juraj Herz semble clairement s'inspirer de ce genre très populaire. Sur toute la longueur de ses 90 minutes, le film reste prisonnier d'une superficialité consternante qui paraît refuser toute allusion trop directe au récit original sur lequel il semble, toutefois, s'appuyer. En revanche, il brille par une distribution impressionnante: Michael Degen en roi père et la vedette de la télévision allemande de l'époque, Iris Berben, dans le rôle de la reine mère. Cependant, aucun message moral n'est identifiable dans ce film. L'acte consistant à lancer la grenouille contre le mur comme élément déclencheur de l'intrigue est simplement supprimé. La question de la moralité de cet acte ne se pose donc même plus. Le prince est un goujat et sa conversion après sa transformation en grenouille et sa re-transformation en jeune homme n'est pas abordée. La princesse, quant à elle, jeune femme indépendante et moderne au début du film - ce qui laissait espérer une histoire d'émancipation - est transformée également à la fin, en fille exemplaire et soumise. Si elle n'évoque guère une princesse au début du film, elle le devient trop à la fin. Si son caractère de garçon manqué au début du film rappelle la merveilleuse Libuše Šafránková de Drei Haselnüsse für Aschenbrödel (réal. : Václav Vorliček), co-production culte des studios tchèques Barrandov et de la DEFA, tournée en 1973, la fille pâle de la fin de l'adaptation n'a plus rien de commun avec cette avant-gardiste 
et féministe comptant parmi les princesses de l'écran. Quant au fidèle Henri, cette version ne se gêne pas non plus pour le supprimer afin de lisser, une fois de plus, son caractère romantique.

Ainsi, au vu de la suppression des deux «blancs » révélateurs de l'intrigue du récit original, on ne peut guère qualifier ce film de réinterprétation ou d'adaptation. L'intrigue du Froschkönig des frères Grimm n'est que la toile de fond d'une comédie romantique à la mode des années 1990.

Avec la dernière adaptation présentée, on revient à une réinterprétation: la princesse de ce conte est réhabilitée. Son accès de colère est, certes, immoral, mais s'explique par son exaspération envers cette pénible grenouille et surtout par un sain esprit de contestation envers un père trop dirigiste et trop protecteur. Quant au fidèle Henri, la plus récente des adaptations propose une représentation classique du fidèle serviteur avec des chaînes autour de la poitrine et un air torturé, mais elle le présente également sous l'aspect mystérieux que suggère le conte littéraire.

Ce film s'inscrit dans le contexte de la pédagogie moderne qui défend la nécessité de révolte de l'enfant face aux parents, surtout si ceuxci, comme le roi-père de cette version, s'avèrent trop protecteurs. La princesse de ce conte apprend à voler de ses propres ailes. Ainsi, c'est surtout le père qui reçoit une leçon: enfermer les enfants pour qu'ils ne puissent s'enfuir ne sert à rien. Il faudrait plutôt leur fournir les moyens de préparer leur départ en paix et avec sérénité. Le cadeau de la mère, symbolisant l'invite à écouter son propre cœur plutôt que d'observer les obligations de la cour, en est le pendant positif. La princesse suit ce conseil posthume de sa mère et jette la grenouille contre le mur. Ce qui est présenté comme un accès de colère injuste dans d'autres versions est ici la réaction compréhensible, voire naturelle d'une jeune femme refusant que d'autres prennent les rênes de sa vie. Quant au fidèle Heinrich, son personnage fait allusion à la mode des romans et films fantastiques. Inspiré par l'amitié, cet homme agit en combattant solitaire et mystérieux, venant des bois brumeux. Son histoire, banale en fin de compte au vu du mystère que le film semble créer autour de lui dès le début, est révélée à la fin du film par le prince délivré. Il s'agit d'une simple histoire d'amitié et de fidélité dont le film ne fait pas grand cas. C'est certainement un de ses défauts principaux : l'histoire du fidèle Heinrich semble quelque peu forcée, « enfoncée » dans l'intrigue.

Mais en somme, cette version, se référant fidèlement au récit original, réussit à concilier les deux exigences d'un bon film de contes de fées: permettre aux jeunes spectateurs de reconnaître le conte original qu'ils ont connu par le récit des frères Grimm et l'actualiser par un message clair et moderne. Le message de cette version est adressé aux enfants et aux parents à la fois, apprenant aux enfants qu'il est normal de se 
révolter contre un excès de protection de la part des parents et aux parents de faire confiance à leurs enfants qui grandissent. De plus, le film réussit ce pari tout en utilisant les moyens de publicité et les stimulateurs de vente qui sont à sa disposition: une distribution attrayante (Friedrich von Thun, Richy Müller) des vedettes de la télévision allemande actuelle et une vaste campagne publicitaire pour la série de contes dans laquelle est intégrée Der Froschkönig.

Il semble donc que ce premier des contes du foyer et de l'enfance est un cas d'école pour l'analyse des techniques utilisées dans l'adaptation d'un conte de fées pour un jeune public. Là où le conte n'est pas assez explicite pour une transposition à l'écran, là où la transposition risque de se heurter à certaines incohérences, trois techniques prennent le relais: l'extrapolation, la réécriture et la suppression. S'il n'y a pas de suppression d'éléments gênants (version de 1990), l'intrigue initiale du conte est complétée par un antécédent (version de 1950, version de 2008) ou une fin modifiée (version de la DEFA, 1987), donc des éléments qui fournissent des explications aux «blancs » ou aux prétendues incohérences du texte original. Dans ce travail de réécriture, d'interprétation, de motivation de certaines bribes d'intrigue, les équipes de films agissent comme des auteurs. En réécrivant, ils interprètent et surtout véhiculent le sens précis qu'ils souhaitent donner à leur version du conte. Ainsi, comme dans toute création, des valeurs précises et/ ou une morale à retenir sont véhiculées. Évidemment, ce sens change selon l'époque à laquelle le film est produit, selon l'orientation que veut lui donner le réalisateur, selon les lignes directrices qui déterminent la production. En ce sens, l'histoire des adaptations des films de contes de fées s'inscrit dans la longue histoire de ce genre littéraire en tant que vecteur de transmission de valeurs et de contenus idéologiques. 
